\title{
Twisting neutral particles with electric fields
}

\author{
Niels Geerits (1)* and Stephan Sponar (1) ${ }^{\dagger}$ \\ Atominstitut, Technische Universität Wien, Stadionallee 2, 1020 Vienna, Austria
}

(Received 28 July 2020; revised 14 January 2021; accepted 19 January 2021; published 8 February 2021)

\begin{abstract}
We demonstrate that spin-orbit coupled states are generated in neutral magnetic spin- $1 / 2$ particles traveling through an electric field. The quantization axis of the orbital angular momentum is parallel to the electric field; hence both longitudinal and transverse orbital angular momentum can be created. Furthermore, we show that the total angular momentum of the particle is conserved. Finally we propose a neutron optical experiment to measure the transverse effect.
\end{abstract}

DOI: 10.1103/PhysRevA.103.022205

\section{INTRODUCTION}

Intrinsic orbital angular momentum (OAM) has been observed in free photons [1-3] and electrons [4-6]. Furthermore, extrinsic OAM states have also been observed in neutrons, using spiral phase plates [7] and magnetic gradients [8]. In the latter case spin-orbit coupled states are generated [9]. It has also been demonstrated that magnetic quadrupoles can generate spin-orbit states in neutral spin-1/2 particles [10,11]. The aforementioned methods require a beam with exceptional collimation $\left(0.01^{\circ}-0.1^{\circ}\right.$ divergence $)$ if intrinsic OAM is the goal. Furthermore, the incident particles must be on the optical axis. These two requirements limit the available flux to an impractical level. For this reason intrinsic OAM has not been observed in neutrons to date [12]. The additional quantum degree of freedom offered by OAM provides utility in the realm of quantum information [13-15]. Additionally in neutrons the additional degree of freedom may help improve existing tests of quantum contextuality $[16,17]$. Furthermore, neutrons carrying net OAM may reveal additional information on atomic nuclei in scattering experiments [18].

In this paper we propose a method by which intrinsic spinorbit states can be generated in an arbitrarily collimated beam of neutral spin- $1 / 2$ particles. This removes flux limitations and allows for the construction of spin-orbit optical equipment for neutrons. We show that a static homogeneous electric field polarized along the direction of particle propagation induces longitudinal spin-orbit states, while a transversely polarized electric field generates transverse spin-orbit states. The latter type of OAM has not yet been observed in massive free particles. Furthermore, we confirm previous results that the total angular momentum of a particle is conserved in static electric fields [19]. As shown by Schwinger [20], in an electric field the particle spin couples to the cross product between

\footnotetext{
"niels.geerits@tuwien.ac.at

${ }^{\dagger}$ stephan.sponar@tuwien.ac.at
}

Published by the American Physical Society under the terms of the Creative Commons Attribution 4.0 International license. Further distribution of this work must maintain attribution to the author(s) and the published article's title, journal citation, and DOI. the electric field strength and the particle momentum. Phase shifts due to this coupling have been observed in Schwinger scattering [21-24], the Aharonov-Casher effect [25-27] and in measurements of the neutron electric dipole moment [28-31], where it can be a major systematic effect. In dynamical diffraction from noncentrosymmetric crystals, spin rotations of up to $90^{\circ}$ have been observed $[23,30]$, due to large interplanar fields. Recently the Schwinger coupling has been used to image electric fields with polarized neutrons [32]. However, to date no tests for OAM have been conducted.

\section{THEORETICAL FRAMEWORK}

An observer moving through an electric field, $E$, will experience a magnetic field $B^{\prime}$. In the low-velocity limit when $v \ll c$ the magnetic field can be written as [33]

$$
\vec{B}^{\prime}=\vec{v} \times \frac{\vec{E}}{c^{2}} .
$$

Inversely in the laboratory frame a moving magnetic moment will appear to have a small electric dipole moment $\vec{d}^{\prime}=\frac{\vec{v} \times \vec{\mu}}{c^{2}}$. Hence a spin-1/2 particle with magnetic moment $\vec{\mu}$ experiences a Zeeman shift $\vec{d}^{\prime} \cdot \vec{E}=\vec{\mu} \cdot \vec{B}^{\prime}$ when moving through an electric field. Therefore, the Schrödinger equation is

$$
\left[-\nabla^{2}-\frac{\gamma}{c^{2}} \vec{\sigma} \cdot(\vec{p} \times \vec{E})\right] \psi=\epsilon \psi,
$$

with $\gamma$ the gyromagnetic ratio and $\vec{\sigma}$ the Pauli matrices. The wave function is described by a spinor $\psi=\left(\begin{array}{l}\psi_{+}(x, y, z) \\ \psi_{-}(x, y, z)\end{array}\right)$, where the index \pm refers to the spin state parallel or antiparallel to the $z$ axis, respectively.

\section{A. Transmission geometry: Longitudinal OAM}

First we consider the longitudinal spin-orbit effect. We assume that the extent of the electric field is semi-infinite and that it is parallel to the $z$ axis. Hence the Schrödinger equation can be written as

$$
-\nabla^{2} \psi_{ \pm}+i C\left(\frac{\partial}{\partial y} \pm i \frac{\partial}{\partial x}\right) \psi_{\mp}=\epsilon \psi_{ \pm},
$$


with $C=\frac{\gamma E_{z}}{c^{2}}$. The incident wave is described by $\psi_{ \pm}^{I}=f(r, \phi) e^{-i k z}$. Note that for a nonzero coupling this effect requires the incident wave function to have a transverse momentum component. By applying a Fourier transform over the $x$ and $y$ coordinates the partial differential equation [Eq. (3)] is simplified to a coupled second-order ordinary differential equation:

$$
-\left(\frac{\partial^{2}}{\partial z^{2}}-k_{r}^{2}+\epsilon\right) \hat{\psi}_{ \pm} \mp i C k_{r} e^{\mp i \phi} \hat{\psi}_{\mp}=0 .
$$

Here we have also transformed the equation to cylindrical coordinates with $k_{r}^{2}=k_{x}^{2}+k_{y}^{2}$ and $k_{x} \pm i k_{y}=k_{r} e^{ \pm i \phi}$. It is noteworthy that in the spectral domain the potential $C\left(k_{x} \sigma_{y}+k_{y} \sigma_{x}\right)$ closely resembles that of the magnetic quadrupole in real space. This gives an intuitive reason as to why a static electric field mimics the action of a quadrupole in reciprocal space. Hence an electric field is more effective for large divergences (i.e., large $k_{r}$ ). We diagonalize Eq. (4) by applying a transformation of the form $\hat{\psi}=T \hat{\psi}^{\prime}$ and multiplying the Hamiltonian by $T^{-1}$ from the left:

$$
\left[-\left(\frac{\partial^{2}}{\partial z^{2}}-k_{r}^{2}+\epsilon\right) \mp C k_{r}\right] \hat{\psi}_{ \pm}^{\prime}=0 .
$$

For this particular diagonalization $T$ is given by $\left(\begin{array}{cc}i e^{-i \phi} & -i e^{-i \phi} \\ 1 & 1\end{array}\right)$. The general solution to Eq. (5) is simply a superposition of a forward- and backward-propagating plane wave for each spin state,

$$
\hat{\psi}^{\prime}=\left(\begin{array}{c}
\hat{t}_{1} e^{i k_{+} z}+\hat{t}_{2} e^{-i k_{+} z} \\
\hat{t}_{3} e^{i k_{-} z}+\hat{t}_{4} e^{-i k_{-} z}
\end{array}\right)
$$

with $k_{ \pm}=\sqrt{\epsilon-k_{r}^{2} \pm C k_{r}}$. Amplitudes of the backwardpropagating solutions, $\hat{t}_{1}$ and $\hat{t}_{3}$, are zero. The general solution for $\hat{\psi}$ is simply found by applying the transformation $T \hat{\psi}^{\prime}$ :

$$
\hat{\psi}=\left(\begin{array}{c}
i e^{-i \phi}\left[\hat{t}_{2} e^{-i k_{+} z}-\hat{t}_{4} e^{-i k_{-} z}\right] \\
\hat{t}_{2} e^{-i k_{+} z}+\hat{t}_{4} e^{-i k_{-} z} .
\end{array}\right)
$$

To determine the values of $\hat{t}_{2}$ and $\hat{t}_{4}$ and the reflection coefficients $\hat{r}_{ \pm}$we apply the boundary conditions

$$
\begin{aligned}
\hat{\psi}\left(k_{r}, \phi, z=0\right) & =\hat{f}_{ \pm}+\hat{r}_{ \pm}, \\
\hat{\psi}_{z}\left(k_{r}, \phi, z=0\right) & =i k_{z}\left(\hat{r}_{ \pm}-\hat{f}_{ \pm}\right) .
\end{aligned}
$$

Here the subscript $z$ under $\psi$ denotes the partial derivative to the $z$ coordinate. $\hat{f}_{ \pm}\left(k_{r}, \phi\right)$ denotes the two-dimensional Fourier transform of the incident wave function. This boundary value problem can be formulated as the following matrix vector problem:

$$
\left(\begin{array}{cccc}
1 & -1 & 1 & 0 \\
1 & 1 & 0 & -1 \\
k_{+} & -k_{-} & -k_{z} & 0 \\
k_{+} & k_{-} & 0 & k_{z}
\end{array}\right)\left(\begin{array}{c}
\hat{t}_{2} \\
\hat{t}_{4} \\
i \hat{r}_{+} e^{i \phi} \\
\hat{r}_{-}
\end{array}\right)=\left(\begin{array}{c}
-i \hat{f}_{+} e^{i \phi} \\
\hat{f}_{-} \\
-i k_{z} \hat{f}_{+} e^{i \phi} \\
k_{z} \hat{f}_{-}
\end{array}\right)
$$

By inverting the above $4 \times 4$ matrix we find the transmission and reflection coefficients,

$$
\begin{aligned}
\hat{t}_{\left(\begin{array}{l}
2 \\
4
\end{array}\right)} & =\frac{\mp i k_{z} \hat{f}_{+} e^{i \phi}+k_{z} \hat{f}_{-}}{\left(k_{z}+k_{ \pm}\right)}, \\
\hat{r}_{ \pm} & = \pm \frac{\left(k_{z}^{2}-k_{+} k_{-}\right) \hat{f}_{ \pm} \mp i k_{z}\left(k_{+}-k_{-}\right) e^{\mp i \phi} \hat{f}_{\mp}}{\left(k_{+}+k_{z}\right)\left(k_{-}+k_{z}\right)},
\end{aligned}
$$

which leads us to the solution for the transmitted waves:

$$
\hat{\psi}_{ \pm}=\frac{k_{z} \hat{f}_{ \pm} \pm i k_{z} \hat{f}_{\mp} e^{\mp i \phi}}{\left(k_{z}+k_{+}\right)} e^{-i k_{+} z}+\frac{k_{z} \hat{f}_{ \pm} \mp i k_{z} \hat{f}_{\mp} e^{\mp i \phi}}{\left(k_{z}+k_{-}\right)} e^{-i k_{-} z} .
$$

Looking at this expression we can see that the total angular momentum $J=S+L$ of the wave is conserved in a static electric field, since a spin flip is compensated by a change in OAM.

$\hat{f}_{ \pm}\left(k_{r}, \phi\right)$ can be expanded such that $\hat{f}_{ \pm}\left(k_{r}, \phi\right)=$ $\sum_{\ell} \hat{f}_{ \pm}^{\ell}\left(k_{r}\right) e^{i \ell \phi}$, where $\hat{f}_{ \pm}^{l}\left(k_{r}\right)$ is given by the azimuthal Fourier transform:

$$
\hat{f}_{ \pm}^{\ell}=\int_{0}^{2 \pi} \hat{f}_{ \pm}\left(k_{r}, \phi\right) e^{-i \ell \phi} d \phi .
$$

The solution in real space can be obtained by applying the Hankel transform to Eq. (11):

$$
\begin{aligned}
\psi_{ \pm}= & \sum_{\ell} i^{-\ell} k_{z} e^{i \ell \theta} \int_{0}^{\infty}\left[\frac{\hat{f}_{ \pm}^{\ell} \pm i \hat{f}_{\mp}^{\ell \pm 1}}{\left(k_{z}+k_{+}\right)} e^{-i k_{+} z}\right. \\
& \left.+\frac{\hat{f}_{ \pm}^{\ell} \mp i \hat{f}_{\mp}^{\ell \pm 1}}{\left(k_{z}+k_{-}\right)} e^{-i k_{-} z}\right] J_{\ell}\left(k_{r} r\right) k_{r} d k_{r} .
\end{aligned}
$$

It is instructive to look at the solution of Eq. (13) for an incident wave field, $\psi_{ \pm}^{I}$, described by a Bessel beam carrying no OAM, $\psi_{ \pm}^{I}=b_{ \pm} J\left(k_{\rho} r\right) e^{-i k_{z} z}$, with $b_{ \pm}$the amplitude of the up- and down-spin states, respectively, and $k_{\rho}$ the transverse momentum component of the incident wave. Hence $\hat{f}_{ \pm}^{\ell \neq 0}=0$ and $\hat{f}_{ \pm}^{0}\left(k_{r}\right)=b_{ \pm} \frac{\delta\left(k_{r}-k_{\rho}\right)}{k_{r}}$ with $\epsilon=k_{z}^{2}+k_{\rho}^{2}$. In this case the solution is trivial:

$$
\begin{aligned}
& \psi_{ \pm}^{0}=k_{z} b_{ \pm} J_{0}\left(k_{\rho} r\right)\left(\frac{e^{-i \sqrt{k_{z}^{2}+C k_{\rho}} z}}{\left(k_{z}+\sqrt{k_{z}^{2}+C k_{\rho}}\right)}+\frac{e^{-i \sqrt{k_{z}^{2}-C k_{\rho}} z}}{\left(k_{z}+\sqrt{k_{z}^{2}-C k_{\rho}}\right)}\right) \\
& \psi_{ \pm}^{1}= \pm k_{z} b_{\mp} J_{1}\left(k_{\rho} r\right)\left(\frac{e^{-i \sqrt{k_{z}^{2}+C k_{\rho}} z}}{\left(k_{z}+\sqrt{k_{z}^{2}+C k_{\rho}}\right)}-\frac{e^{-i \sqrt{k_{z}^{2}-C k_{\rho}} z}}{\left(k_{z}+\sqrt{k_{z}^{2}-C k_{\rho}}\right)}\right)
\end{aligned}
$$

where $\psi_{ \pm}^{0}$ and $\psi_{ \pm}^{1}$ are the components with and without OAM, respectively, such that $\psi_{ \pm}=\psi_{ \pm}^{0}+e^{\mp i \theta} \psi_{ \pm}^{1}$. For a collimated beam geometry we may use $k_{\rho}=k_{z} \tan (\alpha) \approx k_{z} \alpha$, where $\alpha$ is the beam divergence. Furthermore, if $C k_{\rho}$ is sufficiently small 

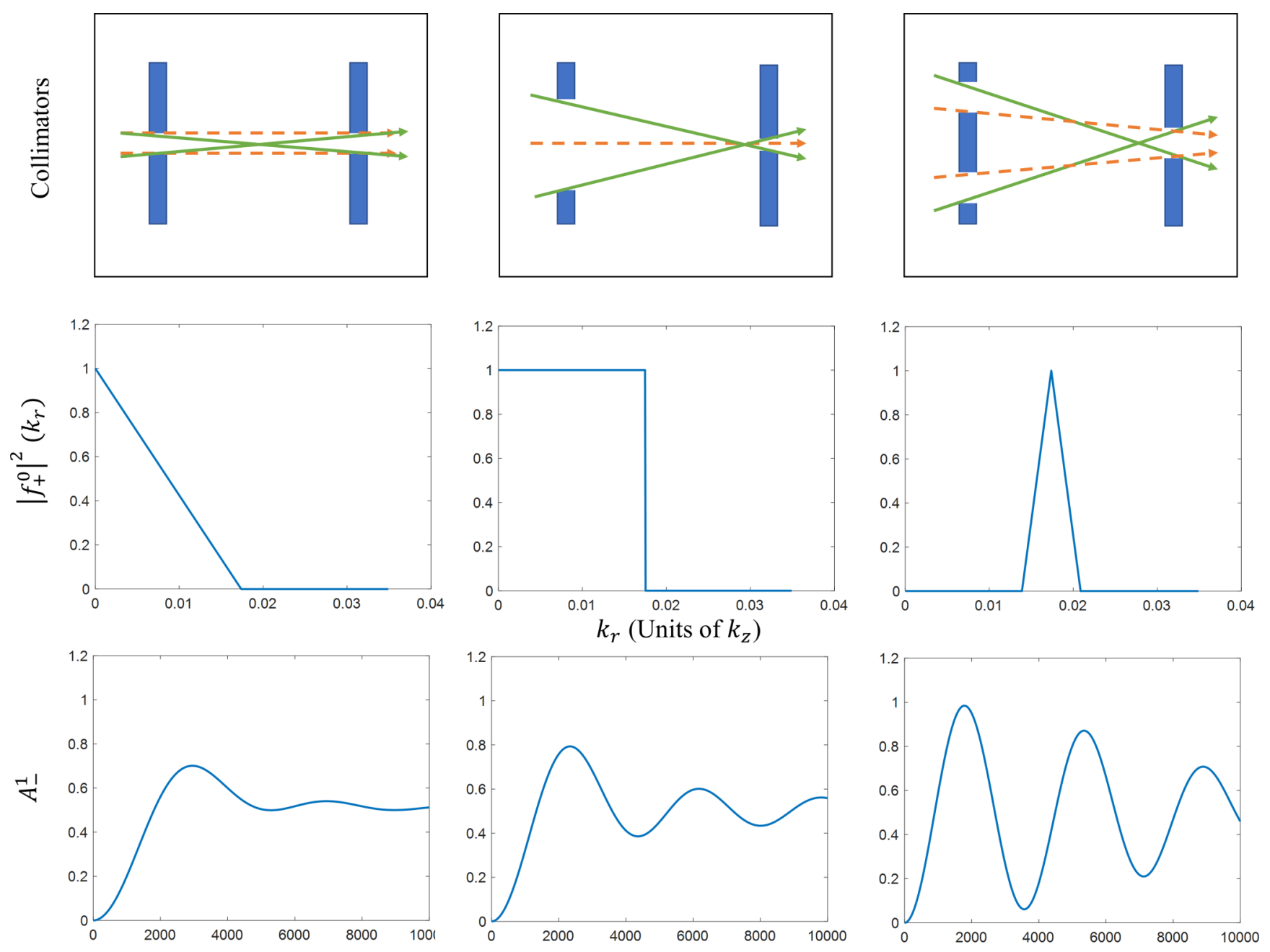

Position (In multiples of wavelength)

FIG. 1. For various common collimator types, such as two identical pinholes (left), a large exit and a comparatively small pinhole (middle), and an annulus with pinhole (right), we show the possible beam paths through a hypothetical instrument (top) and the respective divergence profiles (middle). The paths with the lowest divergence are drawn as dashed orange arrows, while the maximum divergence paths are shown in solid green. The divergence profiles are used as $\left|f_{+}\right|^{2}\left(k_{r}\right)$ in Eq. (17) to determine the probability of finding the particle in the $l=1$ OAM state as a function of the $z$ position in an electric field (bottom). The parameters are chosen such that $k_{z}=1, \epsilon \approx k_{z}^{2}$, and $C=0.1$. We note that in a real instrument these divergence profiles might represent the incoherent average of all possible incident wave fields and not the actual transverse incident wave field of a single neutron.

we may linearize the square-root terms in Eq. (14) and obtain a much simpler expression for the wave function:

$$
\begin{aligned}
\psi_{ \pm}= & {\left[b_{ \pm} \cos \left(\frac{\gamma E_{z} \alpha}{2 c^{2}} z\right) J_{0}\left(k_{\rho} r\right)\right.} \\
& \left. \pm b_{\mp} \sin \left(\frac{\gamma E_{z} \alpha}{2 c^{2}} z\right) e^{\mp i \theta} J_{1}\left(k_{\rho} r\right)\right] e^{-i k_{z} z} .
\end{aligned}
$$

A longitudinal beam twister device may be constructed using a parallel plate capacitor, with the surfaces of the plates normal to the beam. The voltage required to fully twist the beam from the $\ell=0$ state into the $\ell= \pm 1$ state is given by

$$
V=\frac{\pi c^{2}}{\gamma \alpha}
$$

These equations are valid for single Bessel beams. However, Bessel functions are not normalizable [34] and therefore have infinite coherence, making them unphysical. In a realistic setup we always have a normalizable superposition of Bessel beams, which have finite coherence. This superposition interferes and results in damping of spin-orbit production, due to dephasing. This interference can be described by solving Eq. (13) for an arbitrary divergence profile. Though we can also determine the probability of the particle being in the $m$ th OAM state as a function of $z$ without the inverse transform, Eq. (13), by simply calculating the projection of Eq. (11) on $e^{i m \phi}$ and integrating the absolute value squared of this expression over $k_{r}$ :

$$
A_{ \pm}^{m}=\int\left|\hat{\psi}_{ \pm}^{m}\right|^{2} k_{r} d k_{r}=\int\left|\psi_{ \pm}^{m}\right|^{2} r d r
$$

with $\hat{\psi}^{m}=\left\langle e^{i m \phi} \mid \hat{\psi}\right\rangle$, the azimuthal Fourier transform [Eq. (12)] of $\hat{\psi}$. Here we have also used Parseval's theorem to demonstrate that the value of $A^{m}$ is the same in real and 


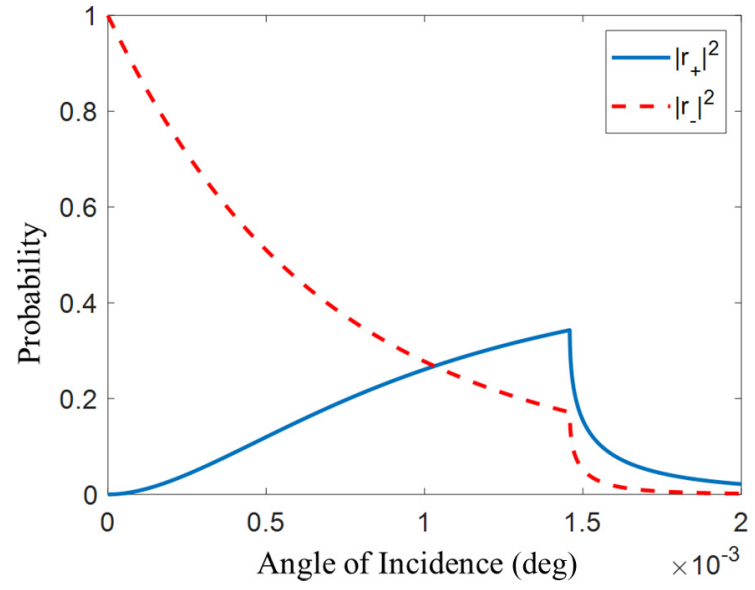

FIG. 2. Reflection probability according to Eq. (10), $\hat{f}_{+}=0$ and $\hat{f}_{-}=1$. A wavelength of $2 \AA$ and an electric field of $10^{10} \mathrm{~V} / \mathrm{m}$ are assumed. The blue solid curve corresponds to a spin-flip reflection which generates OAM, while the red dashed curve shows the nonspin-flip reflection probability.

reciprocal space. Solutions of Eq. (17) for the most common divergence profiles, $|f|^{2}\left(k_{r}, \phi\right)$, are shown in Fig. 1. Here we see dephasing effects, which cause the contrast of $A_{-}^{1}$ to wash out as the wave penetrates deeper into the electric field. As the transverse wavelength spread is decreased, the dephasing effects are also reduced. This is analogous to dephasing seen in magnetic spin-echo instruments due to the longitudinal wavelength spread [35].

Equation (16) demonstrates that for particles with a divergence of $1^{\circ}$ propagating through a capacitor we require a voltage drop of $88.4 \mathrm{GV}$ to put a neutron into an OAM state with $\ell= \pm 1$. Obviously this is not feasible. For colder particles it is possible to use zone plates which consist of concentric rings of periodically spaced absorber material to increase the transverse momentum $k_{r}$, thereby decreasing the required voltage drop. Such Fresnel lenses have been produced for the purpose of imaging with very cold neutrons [36].

\section{B. Reflection geometry: Quasitransverse OAM}

Next we consider waves interacting with an electric field interface at grazing incidence angles. This results in a more pronounced coupling, due to a larger $k_{r}$ and a smaller value for $k_{z}$. The OAM carried by the transmitted and reflected waves in this case is quasitransverse to the wave vector $\vec{k}$. Since the quantization axis of the OAM is normal to the interface, the incident wave must be described by an infinite superposition of OAM modes. Nonetheless, the mean OAM of the transmitted and reflected waves can be raised or lowered by one unit of $\hbar$ with respect to the incident OAM. The reflection probability $\left|r_{ \pm}\right|^{2}$ as a function of incident angle is shown in Fig. 2, for an electric field of $10^{10} \mathrm{~V} / \mathrm{m}$ (found in electric double layers [37,38]), a neutron wavelength of $2 \AA$, and an initial spin aligned along the $-z$ direction. We can deduce that the optimal angle of reflection is around $0.001^{\circ}$. Hence this method of OAM generation is likely not feasible due to flux limitations.

\section{Transmission geometry: Transverse OAM}

The flux limitations can be overcome by considering transmission through a transversely polarized electric field which leads to the generation of transverse spin-orbit states. To demonstrate this we consider the time-dependent Schrödinger equation for a neutral spin-1/2 particle in an electric field:

$$
\left[-\nabla^{2}-\frac{\gamma}{c^{2}} \vec{\sigma} \cdot(\vec{p} \times \vec{E})\right] \psi=-i \frac{\partial}{\partial t} \psi .
$$

Again we assume that the electric field is polarized along the $z$ direction. However, this time we consider a field which extends infinitely in space. To reduce the problem to an ordinary differential equation we apply an unbounded Fourier transform to the spatial coordinates. In cylindrical coordinates this leads to

$$
\epsilon \hat{\psi}_{ \pm} \mp i C k_{r} e^{\mp i \phi} \hat{\psi}_{\mp}=-i \frac{\partial}{\partial t} \hat{\psi}_{ \pm},
$$

where $\epsilon$ now denotes the kinetic energy parameter $k_{r}^{2}+k_{z}^{2}$. Once again we diagonalize this set of equations using the transform $\hat{\psi}=T \hat{\psi}^{\prime}$ :

$$
\left[\epsilon \mp C k_{r}\right] \hat{\psi}_{ \pm}^{\prime}=-i \frac{\partial}{\partial t} \hat{\psi}_{ \pm}^{\prime} .
$$

Applying the initial conditions $\hat{\psi}_{ \pm}(t=0)=\hat{a}_{ \pm}\left(k_{r}, \phi, k_{z}\right)$ we can determine the homogeneous solution of Eq. (19),

$$
\hat{\psi}_{ \pm}=e^{i \epsilon t}\left[a_{ \pm} \cos \left(C k_{r} t\right) \pm a_{\mp} \sin \left(C k_{r} t\right) e^{\mp i \phi}\right],
$$

which appears almost equivalent to Eq. (15). If the wave propagates along the $y$ direction the value of $k_{r}$, which may be approximated by $k_{y}$, is a factor $10^{2}-10^{3}$ larger than in the longitudinal case [Eq. (15)]. Hence the required electric field integral to raise or lower the mean OAM is reduced to a more practical level. The incident wave in this case must be described by an infinite superposition of transverse OAM modes. Upon being transmitted through an ideal beam twister device the mean $\ell$ value of this superposition will be raised or lowered by 1 . In this paper we assume that $\hat{a}_{ \pm}$can be approximated by a Gaussian model. The standard deviation in the $k_{x}$ direction can be expressed in terms of a symmetry factor $R$ and the standard deviation in the $k_{y}$ direction by $\sigma_{y}$ : $\sigma_{x}=R \sigma_{y}$, such that $\hat{a}_{ \pm}=e^{-\frac{\left(k_{y}-k_{y}^{\prime}\right)^{2}}{\sigma_{y}^{2}}} e^{-\frac{k_{x}^{2}}{R^{2} \sigma_{y}^{2}}}$, with $k_{y}^{\prime}$ the mean momentum in the $y$ direction. This Gaussian can be expanded in its various OAM components by means of the azimuthal Fourier transform. Upon passing through an appropriate electric field the index $\ell$ is raised or lowered by 1 . Using this and Eq. (17) the amplitude of the $\ell=1$ OAM mode, $A^{1}$, can be calculated. We may also define an OAM bandwidth in terms of the standard deviation

$$
\sigma_{\ell}=\sqrt{\left\langle L_{z}^{2}\right\rangle-\left\langle L_{z}\right\rangle^{2}}
$$

with $\left\langle L_{z}\right\rangle=\sum_{\ell} \ell A^{\ell}$ and $\left\langle L_{z}^{2}\right\rangle=\sum_{\ell} \ell^{2} A^{\ell}$. Both the OAM amplitude $A^{1}$ and the OAM bandwidth $\sigma_{\ell}$ are shown as a function of the reciprocal longitudinal coherence length $\sigma_{y}$ and the symmetry factor $R$ in Fig. 3. One can see that a small coherence length (large $\sigma_{y}$ ) leads to a larger amplitude $A^{1}$ and a tighter bandwidth $\sigma_{\ell}$. Analogously a large symmetry factor $R$ (i.e., a large beam divergence) corresponds to a larger amplitude $A_{1}$ and a small bandwidth $\sigma_{\ell}$. 
(a)

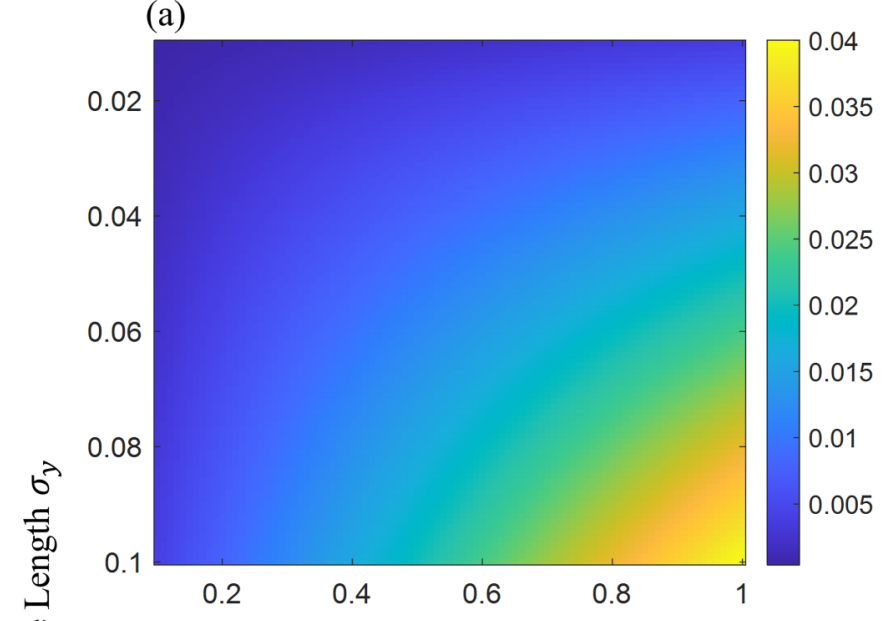

(b)

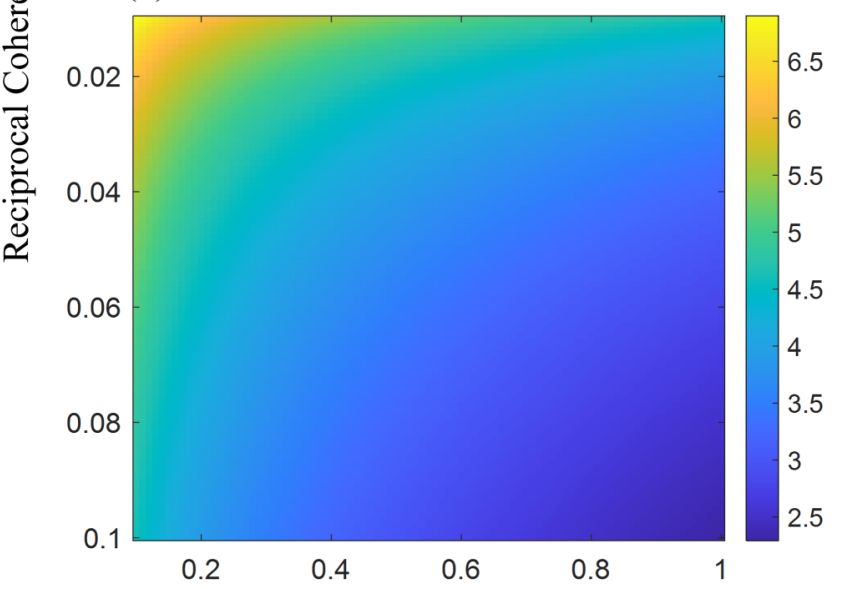

Symmetry Factor R

FIG. 3. (a) The amplitude of the first OAM mode $A^{1}$ and (b) the logarithm of the OAM bandwidth $\sigma_{\ell}$ of a twisted Gaussian wave packet plotted as a function of the reciprocal coherence length $\sigma_{y}$ and the symmetry factor $R$, assuming $k_{y}^{\prime}=1$.

In Fig. 4 we show one such Gaussian wave packet carrying transverse OAM in real space. The wave packet with OAM appears to be displaced along the transverse axis, while along the longitudinal axis the wave packet is shifted by $\pi / 2$.

\section{PROPOSED METHODOLOGY}

Based on the previous theoretical analysis we propose a proof-of-concept experiment with neutrons to demonstrate that magnetic neutral spin- $1 / 2$ particles can obtain quanta of transverse OAM when traversing an electric field polarized perpendicular to the flight direction. The beam twister device will consist of a 1-m-long evacuated flight tube loaded with two electrodes $1 \mathrm{~mm}$ apart. A voltage is applied across the electrodes to generate the experimentally highest possible field in a high-vacuum environment $\left(10^{7}-10^{8} \mathrm{~V} / \mathrm{m}\right)$. Such a beam twister can generate an OAM carrying wave with an amplitude between $2 \%$ and $20 \%$. To measure the OAM we propose an experiment similar to that in Ref. [39], which was designed for photons. The experimental setup would

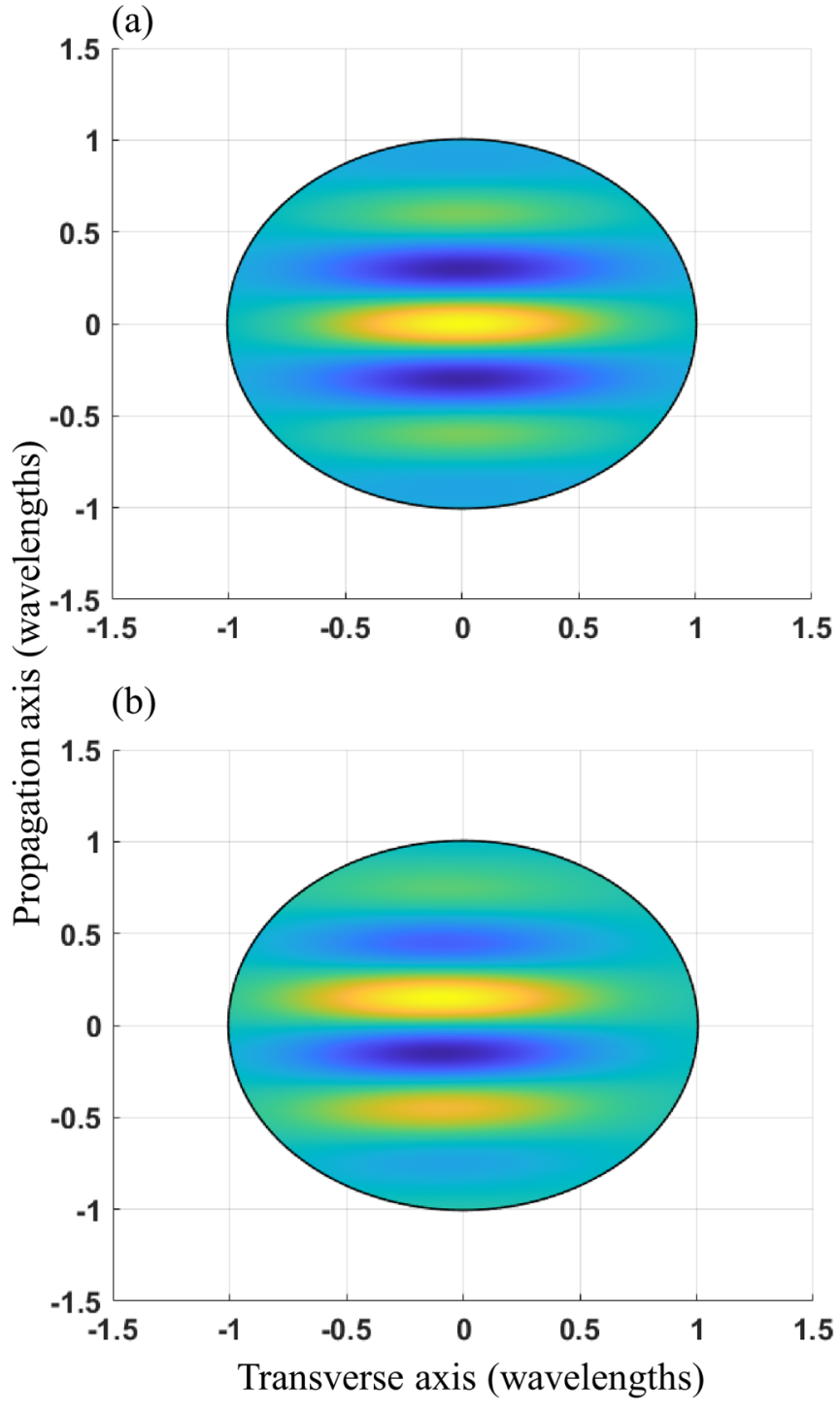

FIG. 4. Surface plots of the real parts of Gaussian wave packets in real space, with $k_{y}^{\prime}=1, \sigma_{y}^{2}=0.1$, and $R=1$ carrying (a) no orbital angular momentum and (b) one unit of transverse orbital angular momentum.

employ two supermirrors to spin-polarize and analyze the beam, two beam twisters to generate and analyze spin-orbit coupling, and a set of three mirrors in between the two beam twisters as a means of rotating the image and inverting the OAM quantum number. This image rotation implies that the quantization axis of the transverse OAM is rotated around the propagation axis. If the dove prism is positioned such that the OAM is flipped the second beam twister will fail to properly decouple the spin-orbit states, thereby leading to destructive interference at the detector. On the other hand, the prism may also be rotated into a position which does not alter the OAM. In this case the second beam twister successfully decouples the neutron spin-orbit states and constructive interference is seen at the detector. Hence by rotating the dove prism at a constant frequency a time-dependent modulation will be seen in the neutron intensity. Since the effects of all components described in this setup are wavelength 


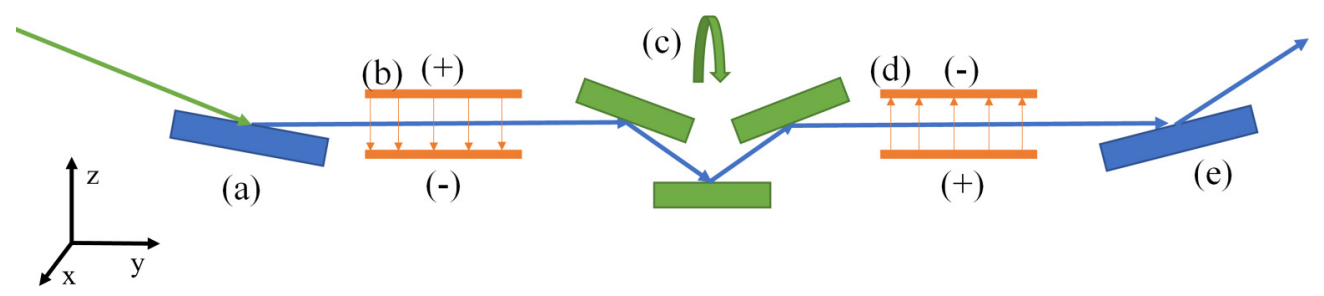

FIG. 5. Simplified schematic of the proposed proof-of-concept experiment to demonstrate the generation of transverse OAM by electric fields. The setup consists of (a) a polarizing supermirror, (b) an electric beam twister, (c) a set of nonpolarizing mirrors which can be rotated around a common beam axis, (d) another beam twister with opposite polarity, and (e) a supermirror for spin polarization analysis.

independent, the experiment can exploit the high thermal flux of a white neutron beam. The proposed setup is shown in Fig. 5.

\section{CONCLUSION}

We have provided a theoretical framework which predicts that magnetic neutral spin-1/2 particles propagating through a static electric field acquire OAM parallel to the electric field axis. Furthermore, we have illustrated a proof-of-concept experiment which could verify the generation of transverse OAM in neutrons transmitted through an electric field.

\section{ACKNOWLEDGMENTS}

The authors thank V. de Haan for fruitful discussion. Furthermore, we would like to extend our gratitude to A. Afanasev for checking the mathematical derivations in this paper. This work was financed by the Austrian Science Fund (FWF), Projects No. P 30677 and No. P 34239.
[1] L. Allen, M. W. Beijersbergen, R. J. C. Spreeuw, and J. P. Woerdman, Orbital angular momentum of light and the transformation of Laguerre-Gaussian laser modes, Phys. Rev. A 45, 8185 (1992).

[2] S. J. van Enk and G. Nienhuis, Spin and orbital angular momentum of photons, Europhys. Lett. 25, 497 (1994).

[3] G. Molina-Terriza, J. P. Torres, and L. Torner, Twisted photons, Nat. Phys. 3, 305 (2007).

[4] B. J. McMorran, A. Agrawal, I. M. Anderson, A. A. Herzing, H. J. Lezec, J. J. McClelland, and J. Unguris, Electron vortex beams with high quanta of orbital angular momentum, Science 331, 192 (2011).

[5] G. Guzzinati, L. Clark, A. Béché, and J. Verbeeck, Measuring the orbital angular momentum of electron beams, Phys. Rev. A 89, 025803 (2014).

[6] V. Grillo, E. Karimi, G. C. Gazzadi, S. Frabboni, M. R. Dennis, and R. W. Boyd, Generation of Nondiffracting Electron Bessel Beams, Phys. Rev. X 4, 011013 (2014).

[7] C. W. Clark, R. Barankov, M. G. Huber, M. Arif, D. G. Cory, and D. A. Pushin, Controlling neutron orbital angular momentum, Nature 525, 504 (2015).

[8] D. Sarenac, C. Kapahi, W. Chen, C. W. Clark, D. G. Cory, M. G. Huber, I. Taminiau, K. Zhernenkov, and D. A. Pushin, Generation and detection of spin-orbit coupled neutron beams, Proc. Natl. Acad. Sci. USA 116, 20328 (2019).

[9] D. Sarenac, J. Nsofini, I. Hincks, M. Arif, C. W. Clark, D. G. Cory, M. G. Huber, and D. A. Pushin, Methods for preparation and detection of neutron spin-orbit states, New J. Phys. 20, 103012 (2018)

[10] E. A. Hinds and C. Eberlein, Quantum propagation of neutral atoms in a magnetic quadrupole guide, Phys. Rev. A 61, 033614 (2000).

[11] J. Nsofini, D. Sarenac, C. J. Wood, D. G. Cory, M. Arif, C. W. Clark, M. G. Huber, and D. A. Pushin, Spin-orbit states of neutron wave packets, Phys. Rev. A 94, 013605 (2016).
[12] R. Cappelletti, T. Jach, and J. Vinson, Intrinsic Orbital Angular Momentum States of Neutrons, Phys. Rev. Lett. 120, 090402 (2018).

[13] G. Vallone, V. D’Ambrosio, A. Sponselli, S. Slussarenko, L. Marrucci, F. Sciarrino, and P. Villoresi, Free-Space Quantum Key Distribution by Rotation-Invariant Twisted Photons, Phys. Rev. Lett. 113, 060503 (2014).

[14] R. Fickler, R. Lapkiewicz, M. Huber, M. P. J. Lavery, M. J. Padgett, and A. Zeilinger, Interface between path and orbital angular momentum entanglement for high-dimensional photonic quantum information, Nat. Commun. 5, 4502 (2014).

[15] D.-S. Ding, M.-X. Dong, W. Zhang, S. Shi, Y.-C. Yu, Y.-H. Ye, G.-C. Guo, and B.-S. Shi, Broad spiral bandwidth of orbital angular momentum interface between photon and memory, Commun. Phys. 2, 100 (2019).

[16] Y. Hasegawa, R. Loidl, G. Badurek, K. Durstberger-Rennhofer, S. Sponar, and H. Rauch, Engineering of triply entangled states in a single-neutron system, Phys. Rev. A 81, 032121 (2010).

[17] J. Shen, S. J. Kuhn, R. M. Dalgliesh, V. O. de Haan, N. Geerits, A. A. M. Irfan, F. Li, S. Lu, S. R. Parnell, J. Plomp, A. A. van Well, A. Washington, D. V. Baxter, G. Ortiz, W. M. Snow, and R. Pynn, Unveiling contextual realities by microscopically entangling a neutron, Nat. Commun. 11, 930 (2020).

[18] A. V. Afanasev, D. V. Karlovets, and V. G. Serbo, Schwinger scattering of twisted neutrons by nuclei, Phys. Rev. C 100, 051601(R) (2019).

[19] S. A. Bruce and J. F. Diaz-Valdes, Neutron interaction with electromagnetic fields: A didactic approach, Eur. J. Phys. 41, 045402 (2020).

[20] J. Schwinger, On the polarization of fast neutrons, Phys. Rev. 73, 407 (1948).

[21] C. G. Shull and R. P. Ferriert, Electronic and Nuclear Polarization in Vanadium by Slow Neutron Scattering, Phys. Rev. Lett. 10, 295 (1963). 
[22] C. G. Shull, Neutron Spin-Neutron Orbit Interaction with Slow Neutrons, Phys. Rev. Lett. 10, 297 (1963).

[23] V. V. Voronin, E. G. Lapin, S. Yu. Semenikhin, and V. V. Fedorov, Depolarization of a neutron beam in Laue diffraction by a noncentrosymmetric crystal, J. Exp. Theor. Phys. 72, 308 (2000).

[24] T. R. Gentile, M. G. Huber, D. D. Koetke, M. Peshkin, M. Arif, T. Dombeck, D. S. Hussey, D. L. Jacobson, P. Nord, D. A. Pushin, and R. Smither, Direct observation of neutron spin rotation in Bragg scattering due to the spin-orbit interaction in silicon, Phys. Rev. C 100, 034005 (2019).

[25] Y. Aharonov and A. Casher, Topological Quantum Effects for Neutral Particles, Phys. Rev. Lett. 53, 319 (1984).

[26] A. Cimmino, G. I. Opat, A. G. Klein, H. Kaiser, S. A. Werner, M. Arif, and R. Clothier, Observation of the Topological Aharonov-Casher Phase Shift by Neutron Interferometry, Phys. Rev. Lett. 63, 380 (1989).

[27] A. Cimmino, B. E. Allman, A. G. Klein, H. Kaiser, and S. A. Werner, High precision measurement of the topological Aharonov-Casher effect with neutrons, Nucl. Instrum. Methods Phys. Res. A 440, 579 (2000).

[28] W. B. Dress, P. D. Miller, J. M. Pendlebury, P. Perrin, and N. F. Ramsey, Search for an electric dipole moment of the neutron, Phys. Rev. D 15, 9 (1977).

[29] P. G. Harris, C. A. Baker, K. Green, P. Iaydjiev, S. Ivanov, D. J. R. May, J. M. Pendlebury, D. Shiers, K. F. Smith, M. van der Grinten, and P. Geltenbort, New Experimental Limit on the Electric Dipole Moment of the Neutron, Phys. Rev. Lett. 82, 904 (1999).

[30] V. V. Fedorov, M. Jentschel, I. A. Kuznetsov, E. G. Lapin, E. Lelièvre-Berna, V. Nesvizhevsky, A. Petoukhov, S. Yu. Semenikhin, T. Soldner, V. V. Voronin, and Yu. P. Braginetz,
Measurement of the neutron electric dipole moment via spin rotation in a non-centrosymmetric crystal, Phys. Lett. B 694, 22 (2010).

[31] F. M. Piegsa, New concept for a neutron electric dipole moment search using a pulsed beam, Phys. Rev. C 88, 045502 (2013).

[32] Y. Y. Jau, D. S. Hussey, T. R. Gentile, and W. Chen, Electric Field Imaging Using Polarized Neutrons, Phys. Rev. Lett. 125, 110801 (2020).

[33] A. Zangwill, Modern Electrodynamics (Cambridge University Press, New York, 2013).

[34] K. Y. Bliokh, I. P. Ivanov, G. Guzzinati, L. Clark, R. Van Boxem, A. Béché, R. Juchtmans, M. A. Alonso, P. Schattschneider, F. Nori, and J. Verbeeck, Theory and applications of free-electron vortex states, Phys. Rep. 690, 1 (2017).

[35] F. Mezei, The principles of neutron spin echo, in Neutron Spin Echo, Lecture Notes in Physics Vol. 128 (Springer, Berlin, 1980), pp. 1-26.

[36] P. D. Kearney, A. G. Klein, G. I. Opat, and R. Gaehler, Imaging and focusing of neutrons by a zone plate, Nature 287, 313 (1980).

[37] M. F. Toney, J. N. Howard, J. Richer, G. L. Borges, J. G. Gordon, O. R. Melroy, D. G. Wiesler, D. Yee, and L. B. Sorensen, Distribution of water molecules at $\mathrm{Ag}(111) /$ electrolyte interface as studied with surface X-ray scattering, Surf. Sci. 355, 326 (1995).

[38] I. Danielewicz-Ferchmin and A. R. Ferchmin, A phase transition in $\mathrm{H}_{2} \mathrm{O}$ due to a high electric field close to an electrode, Chem. Phys. Lett. 351, 397 (2002).

[39] J. Leach, M. J. Padgett, S. M. Barnett, S. Franke-Arnold, and J. Courtial, Measuring the Orbital Angular Momentum of a Single Photon, Phys. Rev. Lett. 88, 257901 (2002). 